\title{
Transcription factors involved in stem cell maintenance are downstream of Slug/Snail2 and repressed by TGF- $\beta$ in bronchial epithelial progenitors from Chronic Obstructive Pulmonary Disease
}

Pierre de la Grange

Genosplice

Ariane Jolly

Genosplice

Charlotte Courageux

INSERM

Chamseddine Ben Brahim

INSERM

Pascale LEROY ( $\nabla$ pascale.leroy@inserm.fr)

https://orcid.org/0000-0001-7635-1358

\section{Research note}

Keywords: COPD, Bronchial epithelium, Primary progenitors, TGF- $\beta$, Slug/Snail2, Somatic stem cell maintenance genes, Transcription factors, Microarray analysis

Posted Date: August 13th, 2019

DOl: https://doi.org/10.21203/rs.2.12678/v1

License: (9) This work is licensed under a Creative Commons Attribution 4.0 International License.

Read Full License 


\section{Abstract}

Objectives: Patients with Chronic Obstructive Pulmonary Disease (COPD) have a bronchial epithelium with many anomalies and basal/progenitor cells showing a decrease of self-renewal and differentiation potential. The objective of this study was to identify deregulations in the genetic program of COPD bronchial progenitors that could account for their exhaustion. The transcription factor Slug/Snail2 is highly expressed in bronchial progenitors and we aimed at identifying genes downstream of Slug whose expression is deregulated in COPD progenitors. Results: We knocked down Slug in primary basal cells from COPD subjects and, since COPD subjects have higher levels of Transforming Growth Factor (TGF)- $\beta$ $0 \mathrm{Cu}$ Slug is regulated by TGF- $\beta$, we selected genes downstream of Slug involved in differentiation that respond to TGF- $\beta$. We identified transcription factors involved in stem cell maintenance downstream of Slug and repressed by TGF- $\beta$ in COPD but not normal progenitors. We found that the effect of TGF- $\beta$ on the expression of these genes is correlated to Slug knockdown effect. We also found a correlation between the mRNA levels of Slug and these genes only in presence of TGF- $\beta$. These results reveal that stem cell maintenance genes are deregulated in COPD bronchial progenitors, Slug and TGF- $\beta$ being involved in that deregulation.

\section{Background}

Subjects with chronic obstructive pulmonary disease (COPD), a respiratory disease mainly caused by cigarette smoke and characterized by a progressive and irreversible loss of respiratory capacity, have a remodeling of their airways with many anomalies of the epithelium that increase with disease progression $(1,2)$. These anomalies are found all along the airway epithelium as cells keep the memory of the exposure to cigarette smoke establishing a "field of injury", and would result from an imbalance of the fate of basal cells, the airway epithelium adult stem/progenitor cells that can self-renew and/or differentiate to repair the epithelium after injury $(3,4)$. A decrease in the count and functional ability of basal progenitors has been reported in smokers with a higher decrease in COPD smokers, leading to a statistically significant difference between non-smokers and smokers-COPD that correlates with the loss of respiratory capacity $(5,6)$.

Slug/Snail2 belongs to the Snail family of transcription factor and is involved in epithelialmesenchymal transition (EMT). In contrast to other EMT-inducing transcription factors, Slug is highly expressed in adult basal/progenitor cells in both mouse and human normal airway epitheliums $(7,8)$. We hypothesized that Slug is involved in the fate of basal/progenitor cells, and, as such, deregulation of its function could lead to the decrease in count and functional ability of progenitors observed in COPD. To test this hypothesis, we exploited the Gene Expression Omnibus (GEO) dataset GSE123129 that we generated from a microarray analysis of Slug knockdown in basal/progenitor cells of COPD and normal subjects (9). Transforming Growth Factor (TGF)-b is found at higher levels in COPD lung tissues and, as it has been shown to regulate Slug expression and to play a role in stem/progenitor cell fate, we also used the GEO dataset GSE122957 to select genes downstream of Slug in COPD that respond to TGF- $\beta$ (9-12). 


\section{Methods}

\section{Study subjects and cell isolation}

Human lung tissues, smokers $(n=6)$ and COPD-smokers $(n=6)$ (see additional file 2) were obtained from patients undergoing lung lobectomy for peripheral lung carcinoma for removal of a primary lung tumor. COPD patients were diagnosed according to the GOLD (Global initiative for chronic Obstructive Lung Disease) guidelines. Lung tissues used in this study were dissected as far away as possible from the tumor. Primary human bronchial epithelial cells were isolated from a piece of large bronchus according to standard protocol.

\section{Cell culture}

Primary human bronchial epithelial cells (Passage 1 or 2 ) were expanded on flasks coated with collagen I (BD Biocoat) in bronchial epithelial growth medium (BEGM), composed of bronchial epithelial basal medium (BEBM) supplemented with the SingleQuots kit (Lonza) and incubated at $37^{\circ} \mathrm{C}$ in $5 \%$ de $\mathrm{CO} 2$. TGF-b1 (Peprotech) was added at a concentration of $1 \mathrm{ng} / \mathrm{ml}$ for 2 days.

RNA extraction

Cells were rinsed with phosphate buffer saline (PBS) before to be homogenized in RNA lysis buffer (NucleoSpin-RNA Kit, Macherey-Nagel), supplemented with 1\% b-Mercaptoethanol. Lysates were vortexed and proceeded immediately or snapped Frozen and stored at $-80^{\circ} \mathrm{C}$. Total RNA was extracted on column using the NucleoSpin-RNA Kit (Macherey-Nagel). DNasel treatment was done directly on the column. RNA concentration was determined using a NanoDrop.

cDNA synthesis

For each sample, $0.3 \mathrm{mg}$ of total RNA was reverse transcribed as previously described (13) with the following modifications: briefly, total RNA was annealed with $0.1 \mathrm{mg} / \mathrm{ml}$ oligo(dT) 15 primer (Promega) and CDNA synthesis was performed with M-MLV Reverse Transcriptase (Promega) for $1 \mathrm{~h}$ at $42^{\circ} \mathrm{C}$. A control without reverse transcriptase was done for each series of cDNAs.

Quantitative Real-Time Polymerase Chain Reaction (qPCR)

Quantitative PCR was done on the QuantStudio6 Flex with the QuantStudio Real-Time PCR software using SYBR green PCR master mix (Applied BioSystems). Primers (see additional file 3) were designed to be used with an annealing temperature of $60^{\circ} \mathrm{C}$ and an elongation time of $1 \mathrm{~min}$. For a given gene target, cDNA volume used was chosen to be in a Ct range from 20 to 30 , using $0.125 \mathrm{mM}$ each forward and reverse primer. Glyceraldehyde-3-Phosphate Dehydrogenase (GAPDH) was used to normalize cDNA amounts between samples.

Gene expression profiling 
Microarray analysis was performed on biological triplicate samples. Total RNA were amplified and labeled before hybridization onto Affymetrix human Gene 2.1ST GeneChip according to the manufacturer, by the Genomics platform at Curie Institute, Paris (14). Array datasets were controlled using Expression console (Affymetrix) and further analyses and visualization were made using EASANA (GenoSplice, www.genosplice.com), which is based on the FAST DB annotations $(15,16)$. Gene Array data were normalized using quantile normalization. Background corrections were made with antigenomic probes and probes were selected as described previously (17). Only probes targeting exons annotated from FAST DB transcripts were selected to focus on well-annotated genes whose mRNA sequences are in public databases (16). Bad-quality selected probes (e.g. probes labeled by Affymetrix as 'cross-hybridizing') and probes whose intensity signal was too low compared to antigenomic background probes with the same GC content were removed from the analysis. Only probes with a DABG P-value $\leq 0.05$ in at least half of the arrays were considered for statistical analysis (17). Only genes expressed in at least one compared condition were analyzed. To be considered expressed, the DABG P-value had to be $\leq 0.05$ for at least half of the gene probes. We performed an unpaired Student's t-test to compare gene intensities in the different biological replicates. Genes were considered significantly regulated when fold-change was $\geq 1.2$ and Pvalue $\leq$ 0.05. Significant KEGG and REACTOME pathways and GO terms were retrieved using DAVID (1820) from union of results of all, up- and down-regulated genes separately. Data set GEO ID numbers are GSE122957 and GSE123129.

\section{Statistical Analysis}

Biological replicates were $n \geq 3$ for the knockdown experiments and $n \geq 5$ for expression analysis with data generated by at least 2 independent experiments. For fold-change, mean is $\pm S E M$ and statistical analysis was carried out by a one sample two-sided t-test. Correlations were computed as Pearson correlation coefficients and P-value determined by two-sided test. Significance was accepted when Pvalue $<0.05$.

\section{Results}

\section{Transcription factor genes involved in somatic stem cell maintenance are repressed downstream of Slug in COPD progenitors}

When setting the minimum fold-change threshold at 1.2 with P-value $\leq 5.00 \mathrm{E}-0,808$ genes were found upregulated in COPD cells knocked down for Slug, i.e. repressed downstream of Slug. Among these, 307 genes are responding to both differentiation and TGF- $\beta$ and we classified them in 4 groups according to their combined type of response. Fig. 1a shows histograms representing the number of genes for each group and small bars indicating the mean fold-increase induced by Slug knockdown for these genes in COPD (red) and normal (blue) cells. The large majority of the genes are upregulated during differentiation and repressed by TGF- $\beta$, and for these the mean fold-increase induced by Slug knockdown is much higher in COPD cells. A search for enriched gene pathways using KEGG, REACTOME and Gene Ontology (GO) databases (18-20) identified 2 pathways related to stem cells, Somatic stem cell population maintenance 
and transcriptional regulation of pluripotent stem cell, comprising 5 genes coding all for transcription factors. We first confirmed by RT-qPCR the fold-change induced by Slug knockdown in COPD and normal cells and Fig. $1 \mathrm{~b}$ shows the correlation with the values obtained in the microarray. The good correlation ( $R^{2}$ values of 0.7309 and 0.5519 , respectively for normal and COPD), validates the microarray analysis and confirms that all the genes are repressed downstream of Slug in COPD cells while they are not downstream of Slug in normal cells, except for ELF5 that is also repressed in normal cells but less than in COPD.

\section{Transcription factor genes involved in stem cell maintenance are more repressed by TGF- $\beta$ in COPD progenitors}

The transcription factor genes involved in stem cell maintenance, which we identified as repressed downstream of Slug in COPD progenitors, were also found by microarray analysis repressed by TGF- $\beta$. We confirmed that all 5 genes are repressed by TGF- $\beta$ in COPD, with the decrease of expression statistically significant except for STAT3. In contrast, different effects, mostly mild, are observed in normal progenitors, none being statistically significant (Fig. 2a). By plotting Slug knockdown effect with that of TGF- $\beta$ on the expression of these genes, we find a good correlation with a $R^{2}$ value of 0.6813 (Fig. $2 b)$. Moreover, for all the genes, except STAT3, a strong negative correlation coefficient is found between their expression levels and that of Slug but only in presence of TGF- $\beta$. The correlation is statistically significant for SOX2 and ELF5 while it is not for EPAS1 and PBX1; however, for these later genes, the correlation strength increases significantly in presence of TGF- $\beta$ (Table1, additional file 1).

Taken together, these results show that in COPD progenitors, when compared to normal, genes coding for transcription factors involved in stem cell maintenance that became direct or indirect repressed targets of Slug, have their response to TGF- $\beta$ deregulated and a link exists between the repressive effect of TGF- $\beta$ and that of Slug.

\section{Discussion}

We identified 5 genes coding for transcription factors involved in somatic stem cell maintenance that are repressed downstream of Slug and by TGF-b in COPD bronchial basal/progenitor cells; in contrast, in normal cells, these genes are less or not repressed by TGF-b, and 4 of them are not downstream of Slug. In COPD, we find a correlation between the effect of Slug knockdown and that of TGF-b on their expression, as well as between their mRNA levels and that of Slug when in presence of TGF-b.

Previous works have shown at the cellular level that bronchial basal/progenitor cells from COPD are deregulated when compared to normal, with a decrease in self-renewal and a loss in differentiation potential leading to progenitor exhaustion $(5,6)$. Our work reveals that key transcription factors involved in stem cell maintenance, direct or indirect repressed targets of Slug, have their expression deregulated in 
COPD, being in particular repressed when in presence of TGF-b. A high level of TGF-b is found in COPD airways and our results are in line with the epithelial progenitor exhaustion described for this disease.

TGF-b represses these transcription factors with different efficiency, and it correlates with the effect of Slug knockdown, showing a link in COPD bronchial progenitors between Slug expression and the response of these genes to TGF-b. This is supported by the high negative correlation coefficient between the mRNA levels of Slug and that of these transcription factors, with the exception of STAT3, found in presence of TGF-b. For EPAS1 and PBX1, despite strong correlation coefficients, P-values are above the significance threshold, showing only a tendency for these genes. However, the small number of patients studied is likely to limit the statistical significance. No such correlation is found for STAT3 but Slug knockdown has the least effect on this gene and it is repressed by TGF-b $\square$ only slightly and with no statistical significance. Each gene have a different profile of regulation by Slug and TGF-b and it is likely that this reflects the existence of other regulations, specific for each gene. TGF-b may coregulate Slug and these genes or, as in other cells, Slug may act as a mediator of TGF-b to regulate these transcription factors $(21,22)$.

Our work reveals that the genetic program of COPD bronchial progenitors is deregulated; this deregulation involves a change in Slug downstream genes and in the response to TGF-b, with consequences on self-renewal and differentiation potential. There is a higher level of TGF-b in COPD and it increases with disease progression (23-25) and we can speculate that progenitor deregulation worsen with the level of TGF-b, participating to COPD progression.

\section{Limitations}

In the present work, the analysis of the stem cell maintenance genes was limited to the

respective effect of Slug knockdown and TGF-b on their expression, and the study of Slug and TGF-b link was limited to expression correlation. Further research on functional analysis is warranted to define the role of these stem cell maintenance transcription factors in bronchial progenitors and the mechanisms involved in their regulation by Slug and TGF-b.

\section{Abbreviations}

COPD Chronic obstructive pulmonary disease, TGF- $\beta$ Transforming growth factor beta, EMT epithelialmesenchymal transition, GAPDH Glyceraldehyde-3-Phosphate Dehydrogenase

\section{Declarations}

\section{Authors' contributions}

PL conceptualized project, conceived and designed the study, conducted experiments, interpreted the data, obtained financial support. PdIG designed the study, analyzed and interpreted the data. AJ 
analyzed the data. CC and CBB performed the experiments. PL and PdIG wrote the manuscript. All authors read and approved the final manuscript.

\section{Author details}

${ }^{1}$ Inserm UMR1152, Physiopathology and Epidemiology of Respiratory Diseases, Paris, France.

${ }^{2}$ Faculty of Medicine, Paris Diderot University, Bichat Campus, Paris, France. ${ }^{3}$ GenoSplice, Paris, France.

\section{Acknowledgements}

We thank the Pulmonary Department, the Pathology Department and the Thoracic Surgery Department at Bichat-Claude Bernard University Hospital (Paris, France) and INSERM UMR 1152 for providing lung tissues and isolating the cells. We thank Audrey Rapinat and David Gentien at the Genomics platform, Curie Institute (Paris, France) for Affymetrix GeneChip hybridization.

\section{Competing interests}

The authors declare that they have no competing interests.

\section{Availability of data and material}

The data analyzed in this publication have been deposited in the NCBI's Gene

Expression Omnibus (GEO) and will be accessible through GEO Series Accession

Number: GSE122957 and GSE123129

\section{Consent for publication}

Not applicable.

\section{Ethics approval and consent to participate}

Human lung tissues were obtained from patients undergoing lung lobectomy for peripheral lung carcinoma after receiving written informed consent. The study was approved by the ethics committee of Paris Nord, IRB 00006477 Paris 7 University, France.

\section{Funding}

P.L. is supported by the French National Center for Scientific Research (CNRS). This work was supported by a donation from Association Science et Technologie (Groupe Servier) to P.L. and by funding from French National Institute for Medical Research (INSERM). The funders had no role in study design, data collection and analysis, interpretation of data and preparation of the manuscript. 


\section{References}

1. Rigden HM, Alias A, Havelock T, O'Donnell R, Djukanovic R, Davies DE, et al. Squamous Metaplasia Is Increased in the Bronchial Epithelium of Smokers with Chronic Obstructive Pulmonary Disease. PLoS One 2016;11(5):e0156009.

2. Sohal SS, Walters EH. Role of epithelial mesenchymal transition (EMT) in chronic obstructive pulmonary disease (COPD). Respir Res 2013;14:120.

3. Rock JR, Randell SH, Hogan BL. Airway basal stem cells: a perspective on their roles in epithelial homeostasis and remodeling. Dis Model Mech 2010;3(9-10):545-56.

4. Steiling K, van den Berge M, Hijazi K, Florido R, Campbell J, Liu G, et al. A dynamic bronchial airway gene expression signature of chronic obstructive pulmonary disease and lung function impairment. Am J Respir Crit Care Med 2013;187(9):933-42.

5. Ghosh M, Miller YE, Nakachi I, Kwon JB, Baron AE, Brantley AE, et al. Exhaustion of Airway Basal Progenitor Cells in Early and Established Chronic Obstructive Pulmonary Disease. Am J Respir Crit Care Med 2018;197(7):885-896.

6. Staudt MR, Buro-Auriemma LJ, Walters MS, Salit J, Vincent T, Shaykhiev R, et al. Airway Basal stem/progenitor cells have diminished capacity to regenerate airway epithelium in chronic obstructive pulmonary disease. Am J Respir Crit Care Med 2014;190(8):955-8.

7. Hackett NR, Shaykhiev R, Walters MS, Wang R, Zwick RK, Ferris B, et al. The human airway epithelial basal cell transcriptome. PLoS One 2011;6(5):e18378.

8. Rock JR, Onaitis MW, Rawlins EL, Lu Y, Clark CP, Xue Y, et al. Basal cells as stem cells of the mouse trachea and human airway epithelium. Proc Natl Acad Sci U S A 2009;106(31):12771-5.

9. Ben Brahim C, Courageux C, Jolly A, Ouine B, Cartier A, de la Grange P, et al. Slug/Snail2 is involved in the repression of proliferation genes by TGF-• in bronchial epithelial progenitor cells and is deregulated in abnormal epithelium. BioRxiv 2019.

10. Mahmood MQ, Reid D, Ward C, Muller HK, Knight DA, Sohal SS, et al. Transforming growth factor (TGF) beta1 and Smad signalling pathways: A likely key to EMT-associated COPD pathogenesis. Respirology 2017;22(1):133-140.

11. Slabakova E, Pernicova Z, Slavickova E, Starsichova A, Kozubik A, Soucek K. TGF-beta1-induced EMT of non-transformed prostate hyperplasia cells is characterized by early induction of SNAI2/Slug. Prostate 2011;71(12):1332-43.

12. Yumoto K, Thomas PS, Lane J, Matsuzaki K, Inagaki M, Ninomiya-Tsuji J, et al. TGF-beta-activated kinase 1 (Tak1) mediates agonist-induced Smad activation and linker region phosphorylation in embryonic craniofacial neural crest-derived cells. J Biol Chem 2013;288(19):13467-80.

13. Leroy $P$, Mostov KE. Slug is required for cell survival during partial epithelial-mesenchymal transition of HGF-induced tubulogenesis. Mol Biol Cell 2007;18(5):1943-52.

14. Maubant S, Tahtouh T, Brisson A, Maire V, Nemati F, Tesson B, et al. LRP5 regulates the expression of STK40, a new potential target in triple-negative breast cancers. Oncotarget 2018;9(32):22586-22604. 
15. de la Grange P, Dutertre $M$, Correa $M$, Auboeuf $D$. A new advance in alternative splicing databases: from catalogue to detailed analysis of regulation of expression and function of human alternative splicing variants. BMC Bioinformatics 2007;8:180.

16. de la Grange P, Dutertre M, Martin N, Auboeuf D. FAST DB: a website resource for the study of the expression regulation of human gene products. Nucleic Acids Res 2005;33(13):4276-84.

17. de la Grange P, Gratadou L, Delord M, Dutertre M, Auboeuf D. Splicing factor and exon profiling across human tissues. Nucleic Acids Res 2010;38(9):2825-38.

18. Haw R, Stein L. Using the reactome database. Curr Protoc Bioinformatics 2012;Chapter 8(Unit8 7.).

19. Huang da W, Sherman BT, Lempicki RA. Systematic and integrative analysis of large gene lists using DAVID bioinformatics resources. Nat Protoc 2009;4(1):44-57.

20. Kanehisa M, Goto S, Sato Y, Furumichi M, Tanabe M. KEGG for integration and interpretation of largescale molecular data sets. Nucleic Acids Res 2012;40(Database issue):D109-14.

21. Geismann C, Arlt A, Bauer I, Pfeifer M, Schirmer U, Altevogt $P$, et al. Binding of the transcription factor Slug to the L1CAM promoter is essential for transforming growth factor-beta1 (TGF-beta)-induced L1CAM expression in human pancreatic ductal adenocarcinoma cells. Int J Oncol 2011;38(1):257-66.

22. Joseph MJ, Dangi-Garimella S, Shields MA, Diamond ME, Sun L, Koblinski JE, et al. Slug is a downstream mediator of transforming growth factor-beta1-induced matrix metalloproteinase-9 expression and invasion of oral cancer cells. J Cell Biochem 2009;108(3):726-36.

23. Godinas L, Corhay JL, Henket M, Guiot J, Louis R, Moermans C. Increased production of TGF-beta1 from sputum cells of COPD: Relationship with airway obstruction. Cytokine 2017;99:1-8.

24. Stoll P, Wuertemberger U, Bratke K, Zingler C, Virchow JC, Lommatzsch M. Stage-dependent association of BDNF and TGF-beta1 with lung function in stable COPD. Respir Res 2012;13:116.

25. Zhang S, Xie JG, Su BT, Li JL, Hu N, Chen J, et al. MFG-E8, a clearance glycoprotein of apoptotic cells, as a new marker of disease severity in chronic obstructive pulmonary disease. Braz J Med Biol Res 2015;48(11):1032-8.

\section{Tables}

Due to technical limitations, Table 1 has been placed in the Supplementary Files section.

\section{Figures}


a

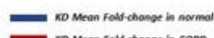

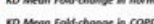

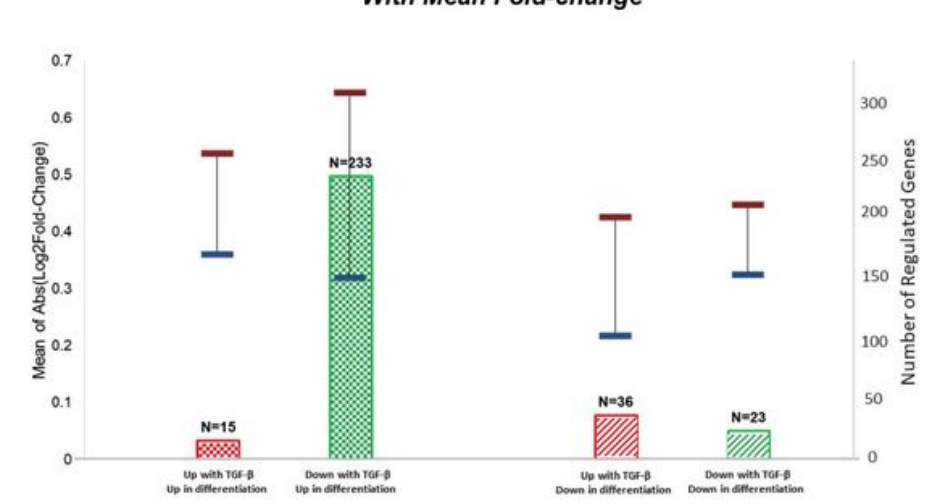

b

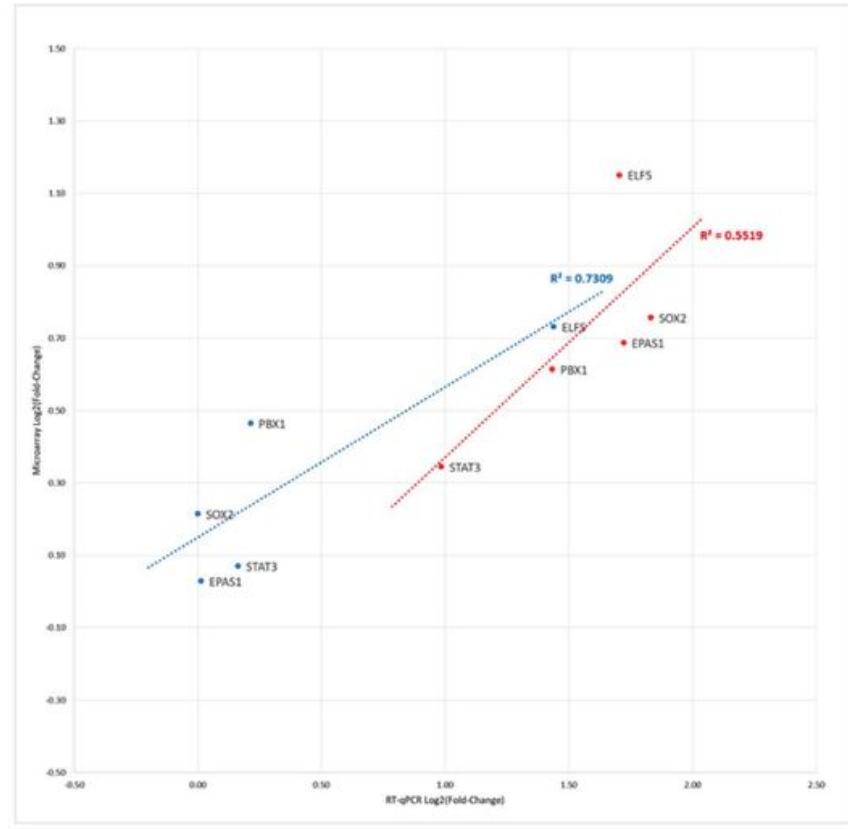

\section{Figure 1}

Slug knockdown in COPD bronchial progenitors identifies repressed genes involved in somatic stem cell maintenance. Primary bronchial epithelial basal cells from COPD and normal subjects were knocked down for Slug, and RNA were analyzed by microarray on an Affymetrix chip. Data are for $n \geq 3$ each, normal and COPD. a Genes significantly upregulated by Slug knockdown in COPD cells and responding to both differentiation and TGF- $\beta$ were classified in 4 groups according to their type of response. Histograms represent the number of genes in each group with genes upregulated by TGF- $\beta$ in red and genes downregulated by TGF- $\beta$ in green, and genes upregulated during differentiation as checkboard and genes downregulated during differentiation as oblique lines. Horizontal bars represent the mean foldincrease for each group of genes, in red for COPD cells and in blue for normal cells. b Validation of microarray by RT-qPCR and comparison of Slug knockdown effect in COPD (red) and normal (blue) cells for the genes coding for 5 transcription factors involved in somatic stem cell maintenance. Results are Pearson correlations calculated with log2 (fold-change) and are presented as scatter plots with regression line and R-square values. 
a

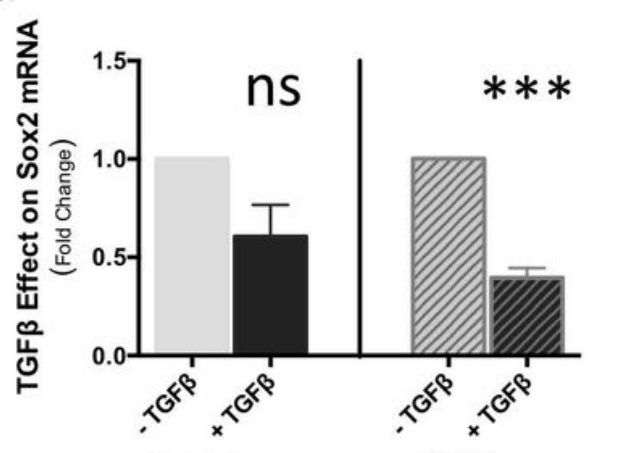

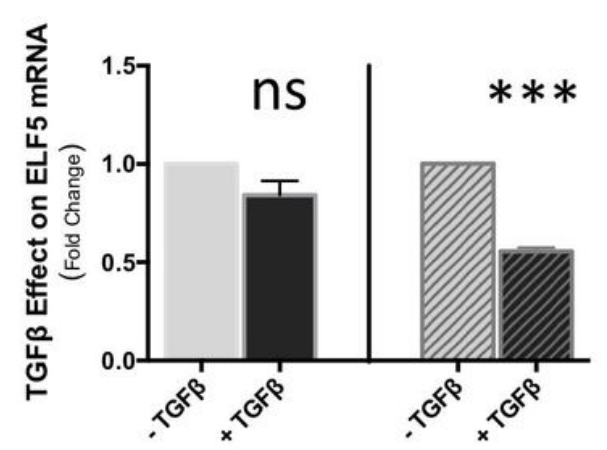

Normal

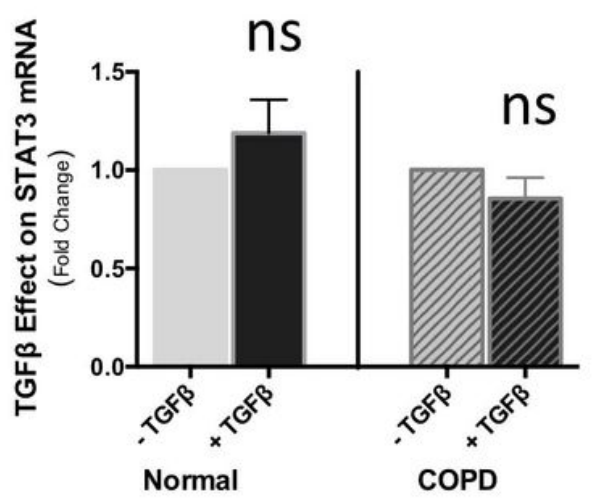

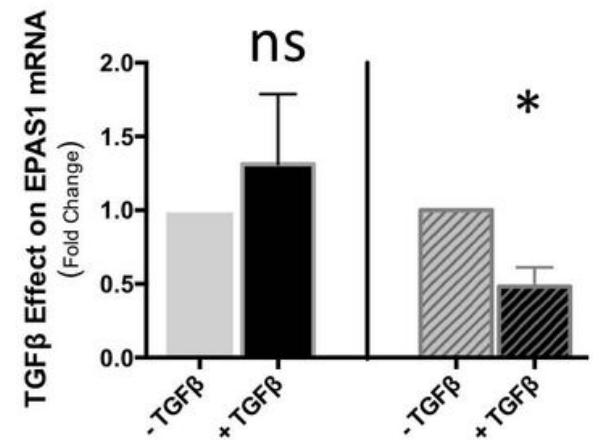

Normal

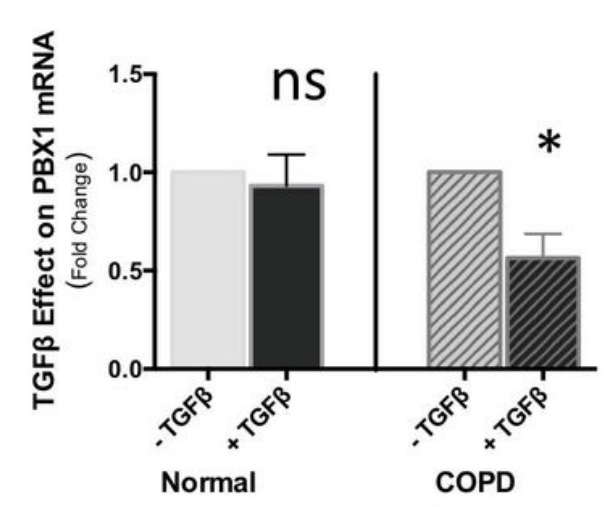

b

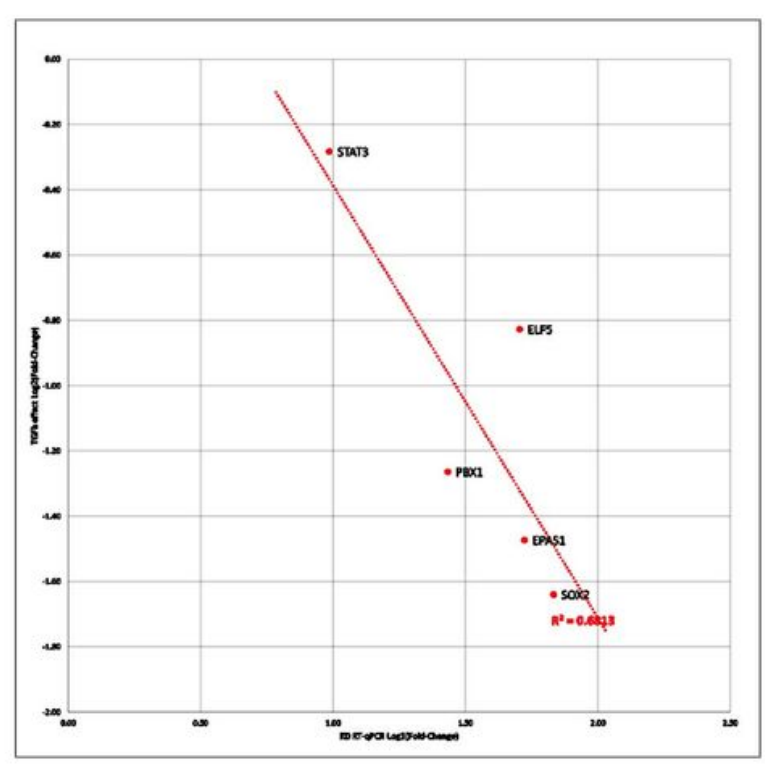

\section{Figure 2}

Genes involved in stem cell maintenance are more repressed by TGF- $\beta$ in COPD bronchial progenitors. Primary bronchial epithelial basal cells, normal and COPD, were grown on filters without TGF- $\beta$ or in presence of $1 \mathrm{ng} / \mathrm{ml}$ of TGF- $\beta$. At confluence, RNA lysates were prepared and mRNA analyzed by RTqPCR. GAPDH was used to normalize cDNA amounts between samples and results were calculated as a ratio on GAPDH. Data shown are for $n \geq 5$. a Comparison of TGF- $\beta$ effect on the expression of stem cell maintenance genes normal and COPD cells. Results are presented as the fold-change induced by TGF- $\beta$ 
on mRNA expression with mean \pm SEM. $b$ Correlation in COPD progenitors between Slug knockdown and TGF- $\beta$ effect on the expression of the 5 genes coding for transcription factors involved in somatic stem cell maintenance. Results are Pearson correlations calculated with log2 (fold-change) and are presented as scatter plots with regression line and R-square values. Statistical significance is at P-value $<5.00 \mathrm{E}-02$ $*_{,}<1.00 \mathrm{E}-03$ *** as indicated. ns: non significant.

\section{Supplementary Files}

This is a list of supplementary files associated with this preprint. Click to download.

- Table1.png

- AdditionalFile2.tif

- AdditionalFile3.tif

- AdditionalFile1.tif 